\title{
Produção Analítico-Comportamental Brasileira sobre Comportamento Matemático e de Ensinar Matemática: Dados de 1970 a 2015
}

\author{
Marcelo Henrique Oliveira Henklain ${ }^{1}$ \\ Universidade Federal de Roraima, Boa Vista, RR, Brasil \\ Universidade Federal de São Carlos, São Carlos, SP, Brasil \\ João dos Santos Carmo \\ Universidade Federal de São Carlos, São Carlos, SP, Brasil \\ Instituto Nacional de Ciência e Tecnologia sobre Comportamento, Cognição e Ensino \\ Verônica Bender Haydu \\ Universidade Estadual de Londrina, Londrina, PR, Brasil
}

\begin{abstract}
Resumo
Os analistas do comportamento têm investigado recursos/estratégias que favorecem o ensino-aprendizagem da Matemática, por exemplo, para o ensino de resolução de problemas, da contagem, e o desenvolvimento de tecnologias para o ensino de pessoas com desenvolvimento atípico. É relevante investir na expansão desses estudos por meio da identificação, organização e sistematização do que já foi produzido. O objetivo deste estudo foi investigar, entre 1970 e 2015, pesquisas brasileiras fundamentadas na Análise do Comportamento sobre os comportamentos matemáticos (CM) e de ensinar Matemática (CEM). Foram consultados sites de programas de pós-graduação e quatro bancos de dados brasileiros: Biblioteca Digital Brasileira de Teses e Dissertações; Biblioteca Virtual em Saúde no Index Psi Teses; Banco de Dados de Trabalhos de Análise do Comportamento; Banco de Teses da Capes. Foram utilizados diferentes descritores a depender da base de dados adotada. Com base na leitura dos resumos, os trabalhos foram selecionados e classificados em CM ou CEM. Os resultados mostraram predomínio de pesquisas sobre CM e a necessidade de mais contribuições sobre CEM. Verificou-se concentração da produção no sudeste, o que indica ser necessário expandir essa fronteira. Pesquisas futuras devem realizar levantamos bibliográficos em artigos nacionais e internacionais sobre o tema.
\end{abstract}

Palavras-chave: Análise do comportamento, ensino-aprendizagem da Matemática, comportamento matemático, ensino de Matemática, levantamento bibliográfico.

\section{Brazilian Behavior-Analytic Literature on Mathematical and Mathematics' Teaching Behavior: Data from 1970 to 2015}

\begin{abstract}
Behavior analysts have investigated resources/strategies that promote the mathematics' teaching and learning, for example, for teaching problem solving, counting, and the development of technologies for teaching people with atypical development. It is important to invest in the expansion of these studies through the identification, organization and systematization of what has been produced. The aim of this study was to investigate, between 1970 and 2015, Brazilian research based on behavior analysis about mathematical behavior (CM) and the behavior of teaching Mathematics (CEM). Graduate programs
\end{abstract}

1 Endereço para correspondência: Rua do Cajueiro, 133, Caçari I, Boa Vista, RR, Brasil 69307-510. Fone: (043) 9938-3704. E-mail: marcelo.henklain@ufrr.br 
sites were consulted and four Brazilian databases: Brazilian Digital Library of Theses and Dissertations; Virtual Health Library in the Index Psi Thesis; Behavior Analysis Studies Database; Capes Thesis Database. Descriptors used were different depending on the database. Based on reading the abstracts, the works were selected and classified into CM or CEM. The results showed a predominance of research on $\mathrm{CM}$ and the need for more contributions about CEM. There was concentration of production in the southeast, which shows that we must expand this boundary. Future research should make bibliographic studies about national and international articles on this subject.

Keywords: Behavior analysis, mathematics teaching and learning, mathematical behavior, mathematics teaching behavior, bibliographical research.

\section{Literatura Analítico-Conductual Brasileña sobre el Comportamiento Matemático y de Enseñar las Matemáticas: Datos de 1970 a 2015}

\section{Resumen}

Los analistas de la conducta han investigado recursos/estrategias que promuevan la enseñanza y la aprendizaje de las matemáticas, por ejemplo, para la enseñanza de la resolución de problemas, la capacidad de contar, y el desarrollo de tecnologías para la enseñanza de las personas con desarrollo atípico. Es importante invertir en la expansión de estos estudios por medio de la identificación, organización y sistematización de lo que se ha producido. El objetivo de este estudio fue investigar, entre 1970 y 2015, la producción brasileña analítico conductual acerca del comportamiento matemático $(\mathrm{CM})$ y de enseñar Matemáticas (CEM). Fueron consultados estudios en los sitios de los programas de posgraduación y en cuatro bancos de datos brasileño: Biblioteca Digital Brasileña de Tesis y Disertaciones; Biblioteca Virtual en Salud (Index Psi Tesis); Banco de Datos de Estudios en la Análisis de la Conducta; Banco de Tesis de la Capes. Descriptores utilizados fueron diferentes en función de la base de datos. Después de la lectura de los resúmenes, las obras seleccionadas fueran clasificadas en CM o CEM. Los resultados mostraron un predominio de CM. Hubo concentración de la producción en la región Sureste. Las investigaciones futuras deberían identificar artículos nacionales e internacionales en esta materia.

Palabras clave: Análisis de la conducta, enseñanza-aprendizaje de matemáticas, comportamiento matemático, comportamiento de enseñanza de las matemáticas, investigación bibliográfica.

O ensino de Matemática é um desafio para pesquisadores e professores, o que se infere com base na associação entre fracasso escolar e dificuldades em Matemática (Araújo \& Luzio, 2005), nos resultados negativos de países em avaliações internacionais (Organization for Economic Cooperation and Development [OEDC], 2012) e no relato de alunos de que a Matemática é uma disciplina difícil e geradora de ansiedade (Mendes \& Carmo, 2014). No Brasil, os resultados que o país tem obtido em avaliações como o Programme for International Student Assessment (PISA) ainda preocupam. Embora tenha sido observada melhora de 356 pontos em 2003 para 391,5 em 2012, o desempenho de alunos do oitavo ano do Ensino Fundamental ao terceiro ano do Ensino Médio nas provas de Matemática ainda foi menor $(391,5)$ do que em leitura (410) ou ciência (405), e o que mais chama a atenção são os dados sobre proficiência em Matemática. Numa escala de sete itens, "abaixo do nível 1", "nível 1", “2”, “3”, “4”, "5" e "nível 6", 35\% dos alunos brasileiros estão classificados como "abaixo do nível 1", 32\% no nível 1 e $20 \%$ no 2. Esse cenário indica que o ensino da Matemática precisa ser aperfeiçoado.

Analistas do comportamento apresentaram diversas contribuições para a Educação em termos filosóficos e de métodos de ensino (Henklain \& Carmo, 2013; Saville, Lambert, \& Robertson, 2011) e especificamente para o ensino de habilidades matemáticas básicas, tais como o conceito 
de número, a resolução de problemas de adição e subtração e o ensino de frações, para diferentes populações, inclusive, pessoas com desenvolvimento atípico (e.g., Escobal, Rossit, \& Goyos, 2010; Lynch \& Cuvo, 1995; Prado \& de Rose, 1999; Santos, Cameschi, \& Hanna, 2012; Santos, Simonassi, Rodrigues, \& Magri, 2014). Diversas pesquisas visaram identificar formas de tornar o aprendizado de Matemática mais fácil e eficaz pela avaliação de diferentes métodos de ensino ou do estabelecimento de comportamento pré-requisito (e.g., Levingston, Neef, \& Cihon, \& 2009; Mayfield \& Chase, 2002) ou, ainda, por meio do desenvolvimento de estratégias específicas, como o emprego de uma balança virtual para resolver problemas de adição e de subtração (e.g., Iégas \& Haydu, 2015). Também têm sido desenvolvidas propostas para o ensino de habilidades complexas como é o caso da Estatística inferencial (e.g., Critchfield \& Fienup, 2010; Fienup \& Critchfield, 2011). Vale destacar, as pesquisas para desenvolvimento de programas/ currículos de ensino de habilidades matemáticas (e.g., Resnick, Wang, \& Kaplan, 1973; Rossit, 2003; Teixeira, 2006).

Os resultados desses estudos relevam a diversidade de problemas sobre ensino-aprendizagem da Matemática que têm sido encaminhados com sucesso por meio dos princípios e métodos analítico-comportamentais. Ao considerar esse potencial e o crescimento experimentado em termos de produção científica, torna-se relevante contribuir com o avanço dessas pesquisas. Isso pode ser feito por meio da reunião e divulgação dos trabalhos desenvolvidos e, sobretudo, da organização desses estudos de modo a contribuir com a elaboração de um programa sistematizado de investigação científica de base comportamental sobre ensino-aprendizagem da Matemática que oriente quais problemas devem ser atacados de forma prioritária e que lacunas precisam ser preenchidas (Carmo \& Prado, 2004).

Este estudo pretende contribuir com a organização e divulgação do conhecimento produzido mediante realização de estudo bibliográfico. Estudos de levantamento bibliográfico contribuem com o avanço da ciência porque organizam e divulgam o conhecimento dispo- nível sobre determinado objeto de estudo num momento específico, destacam as lacunas de conhecimento que precisam ser preenchidas de modo a impulsionar pesquisas empíricas e identificam os principais pesquisadores e centros de pesquisa envolvidos no estudo desse objeto (de Paula, 2009). Esses estudos agilizam o início de novas pesquisas porque diminuem a necessidade de realização de extensos levantamentos bibliográficos a cada nova proposta de investigação, representando uma economia de esforços para outros pesquisadores (Fiorentini, 1994). Os resultados de estudos dessa natureza também têm um valor histórico e representam uma tentativa de preservação da memória de uma área do conhecimento e de avaliação da qualidade da produção científica (Araújo, 2006; Cruz, 2006; Ferreira, 2002).

Uma primeira contribuição envolve organizar a produção da área Comportamental com base nos objetivos das pesquisas. Isso pode ser feito distribuindo as pesquisas em duas classes: estudos sobre "comportamentos matemáticos" (CM) e "comportamentos de ensinar Matemática" (CEM). CM é um conceito que envolve respostas emitidas pelos indivíduos e os estímulos que controlam essas respostas (Carmo \& Prado, 2004; Del Rey, 2009). Para que sejam identificados como "matemáticas", essas respostas e estímulos devem estar relacionados ao que a comunidade verbal classifica como Matemática. Além disso, é possível identificar comportamento matemático com o repertório de respostas, envolvendo números e operações numéricas (Skinner, 1972). Assim, CM pode ser definido como uma relação funcional aprendida entre estímulos socialmente definidos como matemáticos ou propriedades numéricas de eventos ambientais (S) e respostas socialmente definidas como matemáticas ou propriedades de um evento do organismo (R). CEM, por sua vez, abrange os comportamentos de quem ensina, isto é, trata-se do comportamento cuja consequência é o aprendizado de outra pessoa dos conteúdos da Matemática (Teixeira, 2006). Pode ser definido como uma relação funcional entre o comportamento matemático do aprendiz (S) e respostas 
do professor que favoreçam o aprendizado de Matemática pelo aprendiz (R).

Com base na bibliografia revisada (Carmo \& Prado, 2004; Del Rey, 2009; Resnick, Wang, \& Kaplan, 1973; Skinner, 1972), foram criados, para o presente estudo, critérios para classificar uma pesquisa como sendo sobre o "comportamento matemático" (CM) ou sobre o "comportamento de ensinar Matemática" (CEM). Tais critérios foram baseados nos objetivos das pesquisas. Assim, os objetivos de pesquisas sobre CM são: (a) operacionalizar, descrever e analisar funcionalmente comportamentos matemáticos (por exemplo, elaborar a sistematização da rede de relações entre estímulos que compõem o comportamento de somar), (b) verificar a associação entre comportamento matemático e outra variável (por exemplo, evolução das habilidades em Matemática ao longo do Ensino Fundamental) e (c) testar a relação de interferência entre uma variável independente e o comportamento matemático como variável dependente (por exemplo, efeito do ensino de algoritmos sobre o comportamento de resolver problemas de multiplicação). Os objetivos de pesquisas sobre CEM são (a) descrever o comportamento do professor, experimentador ou tutor que ensina Matemática ou elaborar um currículo para o ensino de Matemática (por exemplo, elaborar a sistematização da rede de relações entre estímulos que compõem o comportamento de ensinar um aluno a somar), (b) verificar a associação entre CEM e variáveis dependentes como o tempo de experiência docente e (c) testar os efeitos de uma variável independente sobre o CEM, como variável dependente (por exemplo, efeito do ensino de conceitos de Análise do Comportamento sobre o comportamento de ensinar Matemática).

Um estudo que começou a preencher essa lacuna sobre quais são as contribuições que os analistas do comportamento têm produzido sobre CM foi a dissertação de Del Rey (2009), que investigou essa produção no período de 1970 a 2005. O autor fez o levantamento bibliográfico com base em dissertações e teses. A seleção dos materiais foi feita da seguinte forma: (a) indicação de estudos relacionados a CM por analistas do comportamento; (b) análise dos Currículos Lattes dos autores dos trabalhos indicados para identificação de outros trabalhos; (c) análise das referências dos trabalhos selecionados com o objetivo de encontrar novas indicações de pesquisas. Para cada novo trabalho encontrado, era executado o procedimento de análise das suas referências e do Currículo Lattes dos seus autores; e (d) consulta ao Banco de Dados de Dissertações e Teses em Análise do Comportamento no Brasil 1968-2007 (BDTAC) desenvolvido por Micheletto, Guedes, Pereira e Silva (2008). Ao todo foram selecionadas 24 dissertações e cinco teses.

Del Rey (2009) verificou que no Brasil o estudo dos comportamentos matemáticos foi iniciado nos anos 1970 e que, após 35 anos, foram produzidas 29 dissertações e teses cujos principais temas foram: (a) comportamentos de ordenação; (b) contagem; (c) formação do conceito de número e (d) resolução de problemas. Esses dados corroboram a avaliação de Carmo e Prado (2004) acerca da produção brasileira sobre comportamento matemático. Segundo esses autores, desde os anos 1990 até 2004, os principais temas de pesquisa foram "aquisição do comportamento conceitual numérico" e "aprendizagem da contagem". Uma vez que Del Rey não trabalhou com pesquisas apresentadas em eventos científicos, Henklain e Carmo (2011) buscaram sanar essa lacuna por meio de estudo cujo objetivo foi identificar e descrever, no período de 1992 a 2011, trabalhos de analistas do comportamento sobre ensino-aprendizagem da Matemática apresentados nos Encontros da Associação Brasileira de Psicologia e Medicina Comportamental (ABPMC) e nas Reuniões Anuais de Psicologia da Sociedade Brasileira de Psicologia (SBP). Os autores buscaram os anais e/ou programações desses eventos para identificar trabalhos com, pelo menos, um dos seguintes descritores: (a) número, (b) numeral, (c) Matemática, (d) aritmética, (e) ordenação, (f) quantidade, (g) resolução de problemas. Os resultados indicaram que as pesquisas brasileiras sobre ensino-aprendizagem da Matemática estão concentradas na operacionalização e no ensino de conceitos matemáticos 
básicos, tais como resolução de problemas de soma e subtração, e ensino do comportamento conceitual numérico, na identificação de ansiedade à Matemática e na reversão de dificuldades em relação a essa disciplina.

As pesquisas de Del Rey (2009) e de Henklain e Carmo (2011), foram importantes para iniciar o mapeamento da produção comportamental na área do ensino-aprendizagem da Matemática. Estudos futuros devem buscar identificar publicações de artigos em periódicos científicos e em outros eventos, além dos Encontros da ABPMC e das Reuniões da SBP, o que completaria lacunas deixadas por esses estudos. Além disso, é preciso mapear as pesquisas sobre os comportamentos de ensinar Matemática. Importa lembrar que Del Rey restringiu-se à identificação de pesquisas sobre comportamentos matemáticos. Essas pesquisas contribuem com conhecimentos sobre o que deve ser ensinado nas aulas de Matemática e, naturalmente, são fundamentais. Contudo, esse conhecimento não é suficiente para dar conta do desafio de se ensinar Matemática. Para ser bem sucedido nessa empreitada é preciso também conhecer mais sobre o comportamento de quem ensina essa disciplina.

O objetivo desta pesquisa foi investigar, no período de 1970 a 2015, pesquisas brasileiras (dissertações e teses) fundamentadas na Análise do Comportamento sobre comportamentos matemáticos (incorporando os dados de Del Rey, 2009) e de ensinar Matemática. O período definido se inicia em 1970, ano em que Del Rey identificou o primeiro trabalho sobre CM, e nada foi encontrado a partir da busca feita para a presente pesquisa antes disso; e termina em 2015, ano de submissão deste manuscrito. Na seção de resultados serão usados também os dados de Henklain e Carmo (2011) no intuito de enriquecer a análise e ampliar o escopo deste artigo. A proposta é criar uma discussão direta com os dados já disponíveis na literatura. Em vez de deixar para o leitor buscar estabelecer as relações entre as publicações de Del Rey (2009), Henklain e Carmo (2011), e o presente artigo, foi feita uma análise conjunta de todas essas informações.

\section{Método}

As informações desta seção estão organizadas em diferentes tópicos para facilitar a compreensão das decisões tomadas e suas justificativas. O tópico "Softwares e Organização dos Dados" apresenta como e quais informações foram armazenadas em relação a cada dissertação ou tese selecionada para análise. No tópico "Fontes de Pesquisa" é descrita a estratégia de obtenção de dados e como foram contornadas as limitações encontradas. As principais fontes de pesquisa foram os sites dos programas de pós-graduação e o BDTAC, mas como podiam estar incompletos foram consultadas também bases de dados. O tópico "Expressões de Busca (Descritores)" explica quais descritores foram selecionados e o porquê. Na seção "Procedimento" são fornecidos detalhes sobre como as decisões tomadas nas seções anteriores foram aplicadas para o levantamento das dissertações e teses sobre $\mathrm{CM}$ e CEM.

\section{Softwares e Organização dos Dados}

Foi utilizado o software Microsoft Excel ${ }^{\circledR}$ 2010 para a construção de um banco de dados composto por um conjunto de 24 campos: (a) código do trabalho, (b) nome do autor, (c) título, (d) nível (mestrado ou doutorado), (e) ano de publicação, (f) instituição onde a pesquisa foi realizada, (g) programa de pós-graduação a que o autor estava vinculado na época da pesquisa, (h) agência de fomento, (i) nome do orientador, (j) tipo de pesquisa (histórico-conceitual, básica ou aplicada), (k) delineamento experimental (sujeito único ou grupo), (l) conceitos de Análise do Comportamento utilizados, (m) habilidades matemáticas ensinadas, (n) área da Matemática investigada ou para a qual o trabalho contribuiu, (o) setting ou ambiente em que a pesquisa foi realizada, (p) tipo de desenvolvimento dos participantes (típico ou atípico), (q) idade dos participantes, (r) escolaridade dos participantes, (s) palavras-chave, (t) status (se o trabalho era sobre comportamento matemático ou de ensinar Matemática), (u) fonte na qual o trabalho foi encontrado, (v) link da fonte, (x) link do arquivo com o trabalho completo, (z) resumo. 


\section{Fontes de Pesquisa}

Todos os trabalhos identificados por Del Rey (2009), inclusive sobre CEM, foram armazenados no Banco de Dados de Trabalhos de Análise do Comportamento, BDTAC (Micheletto et al., 2008). Esse banco foi acessado e os trabalhos de Del Rey incorporados ao presente levantamento.

Na pesquisa de Henklain e Carmo (2011), os autores verificaram que as instituições que mais apresentaram trabalhos nas Reuniões Anuais da Sociedade Brasileira de Psicologia (SBP) e nos Encontros da Associação Brasileira de Psicologia e Medicina Comportamental (ABPMC) foram a Universidade Federal de São Carlos - UFSCar (47), a Universidade Federal do Pará - UFPA (26), a Universidade da Amazônia - UNAMA (22), a Universidade Estadual de Londrina - UEL (18), a Universidade Estadual Paulista - UNESP de Marília (15), a Universidade de São Paulo - USP (9) e a Pontifícia Universidade Católica de São Paulo - PUC-SP (7). Del Rey (2009) identificou que UFPA (13), UFSCar (6), USP (4) e UNB (2) destacaram-se mais na produção de dissertações e teses, e indicou quais instituições brasileiras possuem pesquisadores analistas do comportamento que orientam pesquisas sobre ensino-aprendizagem da Matemática. Com base nessas referências, a primeira fonte de dados desta pesquisa foram os Programas de Pós-graduação (PPG) na área de concentração "Psicologia" dessas instituições, a saber: (a) PPG de Psicologia da UFSCar, (b) PPG de Educação Especial da UFSCar, (c) PPG de Análise do Comportamento da UEL, (d) PPG de Psicologia Experimental: Análise do Comportamento da PUC-SP, (e) PPG de Teoria e Pesquisa do Comportamento da UFPA, (f) PPG de Ciências do Comportamento da UNB, (g) PPG em Psicologia da Universidade Federal de Santa Catarina (UFSC), (h) PPG em Psicologia Experimental da USP, (i) PPG em Psicologia da PUC-GO e (j) PPG em Psicologia do Desenvolvimento e Aprendizagem da UNESP-Bauru.

A vantagem de consultar os sites dos programas é que, geralmente, eles relacionam todas as dissertações e teses produzidas desde a criação do programa. Contudo, por reconhecer que os sites poderiam não ter sido atualizados ou que dissertações e teses de interesse para este trabalho poderiam ter sido produzidas em outros programas não selecionados, decidiu-se consultar três bancos de dados brasileiros que disponibilizam dissertações e teses: (a) Biblioteca Digital Brasileira de Teses e Dissertações, BDBTD (http://bdtd.ibict.br/); (b) Biblioteca Virtual em Saúde no Index Psi Teses, BVS-Psi (http://www. bvs-psi.org.br); (c) Banco de Teses da Capes com dados de 2011 e 2012 (http://bancodeteses. capes.gov.br).

\section{Expressões de Busca (Descritores)}

Foram testados diversos descritores em cada base de dados de modo a selecionar aqueles que mais trouxessem resultados em cada contexto de pesquisa, sem a exigência de que um mesmo conjunto de descritores fosse usado em todas as bases. Foram testados os seguintes descritores de forma isolada e combinada: "número", "numeral", "Matemática", "aritmética", "ordenação", "quantidade" e "resolução de problemas", que foram os mesmos adotados por Henklain e Carmo (2011), as expressões "análise do comportamento", "equivalência de estímulos", "Matemática" e "ensino", e termos diretamente relacionados à Matemática, tais como adição, subtração, fração, função do primeiro grau e contagem.

Nas buscas, foi considerado o máximo de variações de cada descritor. Por exemplo, em relação a número e numeral, foram considerados os termos números, numérico e numerais; o descritor Matemática, por sua vez, incluía matemáticas, matemático e matemáticos. Importa ressaltar que nas buscas não foram feitas restrições em relação à localização dos descritores no documento, ou seja, eles poderiam estar no título, no resumo, no texto completo, etc. Os descritores selecionados (incluindo suas variações) para pesquisa nas bases de dados foram "equivalência de estímulos", "análise do comportamento", "Matemática" e "ensino de Matemática". Essa constatação reforça os dados encontrados por Henklain e Carmo (2011) relativos às palavras-chave mais utilizadas nas pesquisas apresentadas na SBP e na ABPMC. Os autores verificaram que dentre 271 palavras 
identificadas, as mais frequentes foram "equivalência de estímulos" (24), "conceito de número" (10), "análise do comportamento" (9), "comportamento matemático" (9) e "contagem" (9). Verificou-se ainda que os trabalhos que usaram palavras como "contagem ou conceito de número", também empregavam a expressão "equivalência de estímulos" ou "Matemática" ou "análise do comportamento".

Nos sites dos programas de pós-graduação foram lidos todos os títulos das teses e dissertações disponíveis e, por esse motivo, foi possível considerar no momento da seleção todos os descritores inicialmente testados (o mesmo procedimento foi adotado na leitura dos trabalhos do BDTAC). Nas bases de dados, a pesquisa ficou organizada do seguinte modo: (a) na BVS-Psi foram utilizados como descritores "análise do comportamento", "equivalência de estímulos" e "Matemática"; (b) na BDBTD foi utilizada uma expressão de busca: + "análise do comportamento" AND matem?tic* AND +comportament* $O R$ ensin*, que obrigava o mecanismo de busca (pelo uso do sinal +) a apresentar resultados com "análise do comportamento" (todos essas palavras juntas por conta do uso das aspas duplas) e termos como "Matemática(s)" ou "matemático(s)", sendo que todas as palavras poderiam aparecer com ou sem acento por conta do uso do sinal de interrogação, "matem?tic", e com ou sem o "s" por conta do uso do asterisco ao final da palavra. Prosseguindo, além desses dois requisitos, os resultados deveriam conter os descritores comportamento ou ensino e suas variações (por exemplo, comportamentos, ensinamento, ensina, etc.); (c) no Banco de Teses da Capes foram utilizados os descritores "equivalência de estímulos", "análise do comportamento" e a expressão "“"ensino de matemática” AND comportamento". O uso do descritor "Matemática" foi evitado porque gerou 3.940 registros, tornando a consulta inviável.

\section{Procedimento}

Os sites dos programas de pós-graduação foram acessados. Apenas os programas das PUC's e da USP redirecionam o usuário para a página da Biblioteca Virtual, de modo que a pes- quisa às dissertações e teses fosse efetuada. Nos dois casos, os títulos dos trabalhos foram lidos na íntegra. Foram selecionadas para leitura do resumo apenas as dissertações e teses que contivessem no título, pelo menos, dois dos seguintes descritores: "análise do comportamento", "equivalência de estímulos", "Matemática" (ou termo diretamente referente à Matemática, tais como adição, subtração, fração, função do primeiro grau, etc.) ou os descritores usados por Henklain e Carmo (2011).

Nos bancos de dados, os descritores foram inseridos nos campos de busca em conformidade com as regras expostas na seção anterior. Os títulos dos trabalhos que apareciam como resultados eram lidos na íntegra. Aqueles que contivessem, pelo menos, dois dos descritores supracitados eram selecionados para o procedimento de leitura do resumo.

Foram considerados trabalhos da abordagem comportamental aqueles que (a) alegaram no resumo filiação a abordagem comportamental ou (b) cuja autoria fosse de um dos analistas do comportamento citados por Del Rey (2009), pois seu procedimento garantiu a verificação da abordagem a que o autor pertencia ou, ainda, que o autor possuísse indicação no seu currículo Lattes de que pesquisa na área de Análise do Comportamento ou (c) que empregasse conceitos comportamentais para a análise dos dados ou (d) que apresentasse no resumo o objetivo de realizar uma análise comportamental.

A classificação das pesquisas quanto ao seu objetivo, CM ou CEM, foi realizada a partir da leitura dos resumos com base nos critérios expostos na introdução deste artigo. Um trabalho sobre CM pode ter por objetivo, por exemplo, ensinar o comportamento de somar. Note que o trabalho não focaliza o comportamento de quem ensina, mas o do aprendiz. Por isso, é classificado como trabalho de CM. Por outro lado, quando a pesquisa define como objetivo analisar o comportamento do professor ou, por exemplo, ensiná-lo a realizar análise funcional para que ele aprimore suas aulas de Matemática, então a ênfase recai sobre o comportamento de quem ensina e não o do aprendiz. Após a leitura dos resumos, os trabalhos que atendiam aos critérios 
expostos foram selecionados para cadastro das informações descritas no tópico "Softwares e Organização dos Dados" e análise.

\section{Resultados}

A busca na BDBTD resultou em 74 trabalhos e na BVS-Psi, cujos descritores usados foram mais amplos, resultou em 324 trabalhos. No Banco de Teses da Capes, foram obtidos 464 trabalhos. No BDTAC foram encontrados seis estudos e nos sites dos programas, considerando todos juntos, foram encontrados 30 trabalhos. $\mathrm{Na}$ época da pesquisa nem todos os sites dos programas estavam com informações completas até 2015, a saber: o Programa de Ciências do Comportamento da Universidade de Brasília estava atualizado até 2014, o Programa de Educação Especial da Universidade Federal de São Carlos até 2013 e o Programa de Pós-graduação em Psicologia da Universidade Federal de Santa Catarina até 2011. Os demais sites relacionavam todas as teses e dissertações defendidas até 2015.
Muitas pesquisas encontradas não passaram pela seleção final, cujo objetivo era definir se o trabalho era sobre Matemática e se adotava a Análise do Comportamento como referencial teórico. Além disso, um mesmo trabalho foi encontrado em mais de uma fonte; geralmente, o que era encontrado no site do programa, também foi identificado nas bases de dados. Ao final desse procedimento de análise, foram selecionados 60 estudos, sendo 10 sobre o comportamento de ensinar Matemática e 50 sobre comportamento matemático (desses 50, 29 foram os trabalhos encontrados por Del Rey, 2009).

A Figura 1 exibe quatro curvas acumuladas: (a) dissertações e teses sobre comportamento matemático (CM), (b) pesquisas sobre comportamento matemático apresentadas nos eventos da ABPMC e SBP, que foram dados apresentados por Henklain e Carmo (2011), (CM-EV), (c) dissertações e teses sobre o comportamento de ensinar Matemática (CEM) e (d) pesquisas sobre o comportamento de ensinar Matemática apresentadas nos eventos da ABPMC e SBP (CEM-EV).

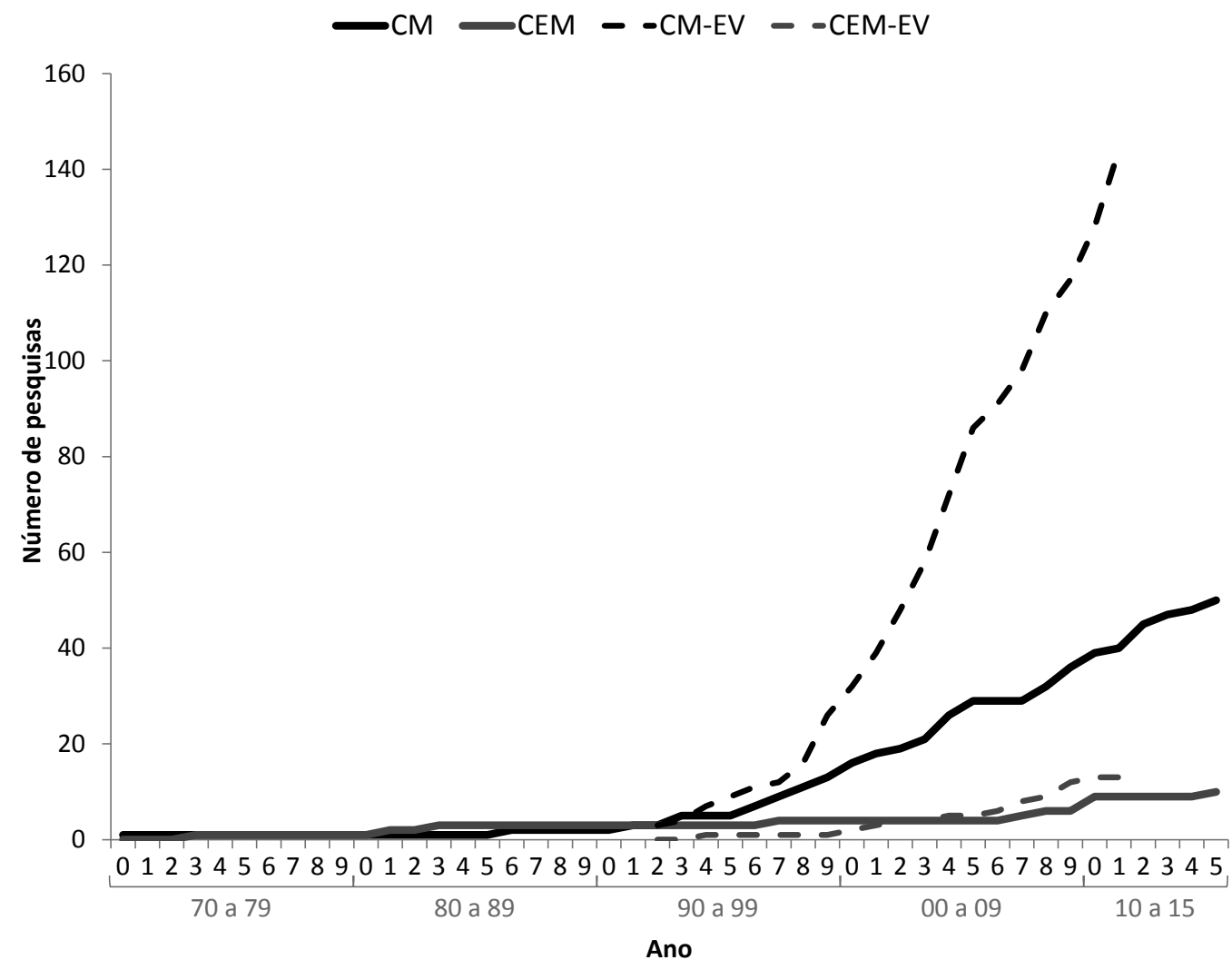

Figura 1. Curva acumulada do número de dissertações e teses, de 1970 a 2015, sobre comportamento matemático (CM) e comportamento de ensinar Matemática (CEM), e pesquisas, curvas tracejadas, apresentadas nos eventos da ABPMC e SBP sobre comportamento matemático (CM-EV) e comportamento de ensinar Matemática (CEM-EV). 
Observa-se na Figura 1 que existe diferença considerável entre CM e CEM, seja em termos de dissertações e teses (50 CM contra $10 \mathrm{CEM}$ ) ou de pesquisas apresentadas em eventos (144 CM contra 13 CEM). Existe uma relação de cinco pesquisas de CM para cada pesquisa de CEM no caso de dissertações e teses, sendo, aproximadamente, o dobro no caso de eventos, $11 \mathrm{CM}$ para cada CEM.

De 1970 a 1995 , poucas pesquisas foram executadas sobre CM (acumulado de cinco trabalhos, ou seja, cerca de 0,2 por ano) ou CEM (três, ou seja, 0,11 por ano) e, nesse período, existia equilíbrio entre a quantidade de pesquisas sobre CM e CEM. A partir de 1996, começam a ser produzidos mais trabalhos sobre CM e a apresentação de trabalhos em eventos, curva CM-EV, acompanha essa tendência. A partir de 1999, ocorre um afastamento acentuado entre dissertações e teses produzidas sobre $\mathrm{CM}$ e os trabalhos apresentados em eventos (o dobro de pesquisas apresentadas em eventos em relação às dissertações e teses). As curvas relativas às pesquisas sobre CM possuem aceleração positi- va, sendo a de CM-EV mais acentuada, ao passo que as curvas de CEM apresentam alguns momentos de pequena aceleração positiva (CEM: 1996, 2007, 2010 e 2014; CEM-EV: entre 2000 e 2002, 2004, entre 2006 e 2010), mas, de modo geral, observa-se que a produção ficou estagnada. Importa acrescentar que existe um número mais expressivo de dissertações do que teses (total: 45 contra 15, respectivamente), sendo 37 dissertações de CM contra 8 de CEM, e 13 teses de CM contra 2 de CEM.

A Tabela 1 exibe a quantidade de dissertações e teses e de pesquisas apresentadas em eventos por instituição, podendo-se observar que a UFPA e a UFSCar se destacam em termos de produção sobre CM e CEM (total de 35 e 45 pesquisas, respectivamente), tanto na dimensão dissertações e teses quanto na dimensão de resumos publicados de trabalhos apresentados em eventos. Com quantidade elevada de produção aparecem UEL (17), PUC-SP (14), USP (11) e UNESP-Bauru (10). A categoria "Outras" foi formada por instituições com menos de quatro trabalhos (dissertação/tese ou pesquisa apresentada em evento).

Tabela 1

Distribuição do Número de Dissertações e Teses e de Pesquisas Apresentadas em Eventos por Instituição e em Função da Pesquisa ser Classificada como CM ou CEM

\begin{tabular}{|c|c|c|c|c|}
\hline \multirow{2}{*}{ Instituições } & \multicolumn{2}{|c|}{ Dissertações \& Teses } & \multicolumn{2}{|c|}{ Pesquisas em Eventos } \\
\hline & $\mathrm{CM}$ & CEM & $\mathrm{CM}$ & CEM \\
\hline UFPA & 17 & 0 & 18 & 0 \\
\hline UFSCar & 11 & 1 & 31 & 2 \\
\hline PUC-SP & 7 & 0 & 7 & 0 \\
\hline USP & 4 & 2 & 5 & 0 \\
\hline UNESP-Bauru & 0 & 6 & 2 & 3 \\
\hline UEL & 4 & 0 & 13 & 0 \\
\hline UNAMA & 0 & 0 & 11 & 1 \\
\hline UNESP-Marília & 0 & 0 & 10 & 1 \\
\hline UFMG & 0 & 0 & 4 & 1 \\
\hline UCG & 0 & 0 & 4 & 0 \\
\hline Outras & 7 & 1 & 39 & 5 \\
\hline Subtotal & 50 & 10 & 144 & 13 \\
\hline Total & \multicolumn{2}{|c|}{60} & \multicolumn{2}{|c|}{157} \\
\hline
\end{tabular}


A Tabela 2 exibe uma relação dos pesquisadores que mais orientaram trabalhos (dissertações e teses) na área e a quantidade de trabalhos orientados. Os principais orientadores são, atualmente, conforme consulta ao Currículo Lattes, professores da UFPA e da UFSCar, mas é preciso ressaltar que a PUC-SP apareceu como uma instituição que reúne diversos pesquisadores envolvidos com estudos sobre ensino-aprendizagem da Matemática, embora no total a produção seja menor que a identificada nas duas primeiras universidades. Os demais trabalhos se distribuem, principalmente, entre orientadores de instituições do estado de São Paulo e do Paraná, no caso, a UEL.

Tabela 2

Distribuição do Número de Dissertações e Teses Orientadas por Pesquisador

\begin{tabular}{|c|c|c|c|c|}
\hline Orientador & Instituição & $\mathrm{CM}$ & CEM & Total \\
\hline Assis, G. J. A. & UFPA & 9 & 0 & 9 \\
\hline Galvão, O. F. & UFPA & 5 & 0 & 5 \\
\hline Carmo, J. S. & UFSCar & 5 & 0 & 5 \\
\hline Bori, C. M. & USP & 2 & 2 & 4 \\
\hline Júnior, J. L. & UNESP-Bauru & 0 & 4 & 4 \\
\hline Goyos, A. C. N. & UFSCar & 4 & 0 & 4 \\
\hline Haydu, V. B. & UEL & 3 & 0 & 3 \\
\hline Matos, M. A. & USP & 1 & 1 & 2 \\
\hline Cavalcante, M. R. & UNESP-Bauru & 0 & 2 & 2 \\
\hline Tourinho, E. Z. & UFPA & 2 & 0 & 2 \\
\hline Medeiros, J. G. & UFSC & 2 & 0 & 2 \\
\hline Luna, S. V. & PUC-SP & 2 & 0 & 2 \\
\hline Botomé, S. P. & UFSC & 0 & 1 & 1 \\
\hline Ferrara, M. L. D. & USP & 1 & 0 & 1 \\
\hline Fontes, J. C. S. & UFPA & 1 & 0 & 1 \\
\hline Hanna, E. S. & UNB & 2 & 0 & 2 \\
\hline Castro Neto, J. M. O. & UNB & 1 & 0 & 1 \\
\hline Souza, D. G. & UFSCar & 1 & 0 & 1 \\
\hline Micheletto, N. & PUC-SP & 1 & 0 & 1 \\
\hline Banaco, R. A. & PUC-SP & 1 & 0 & 1 \\
\hline Moroz, M. & PUC-SP & 1 & 0 & 1 \\
\hline Sério, T. M. A. P. & PUC-SP & 1 & 0 & 1 \\
\hline Souza, S. R. & UEL & 1 & 0 & 1 \\
\hline Pereira, M. E. M. & PUC-SP & 1 & 0 & 1 \\
\hline Rose, J. C. C. & UFSCar & 1 & 0 & 1 \\
\hline Enumo, S. R. F. & UFES & 1 & 0 & 1 \\
\hline Simonassi, L. E. & PUC-GO & 1 & 0 & 1 \\
\hline Total & & 50 & 10 & 60 \\
\hline
\end{tabular}


Foi realizada contagem do número de ocorrências de cada palavra-chave indicada nos trabalhos identificados e as principais foram, novamente, equivalência de estímulos, análise do comportamento e Matemática. Embora sejam descritores bastante genéricos, os dados têm apontado que são os descritores mais comumente empregados pelos pesquisadores da área para caracterizar seus trabalhos. Com efeito, após a inclusão desses descritores, os autores incluem outros mais específicos, tais como "resolução de problemas", "comportamento conceitual numérico", "contagem", entre outros.

\section{Discussão}

O objetivo deste estudo foi realizar um levantamento bibliográfico de dissertações e teses brasileiras de base comportamental no período de 1970 a 2015 acerca do tema comportamentos matemáticos e comportamentos de ensinar Matemática. A significativa diferença de produção entre CM e CEM, atrelada ao potencial contributivo dos estudos existentes sobre CEM, sugere que os analistas do comportamento devem ampliar os estudos sobre CEM, uma vez que um dos aspectos enfatizados por Skinner é a importância que o ensino tem para que ocorra a aprendizagem (Skinner, 1968/1972), ou seja, o comportamento de quem ensina deve ser objeto da atenção dos pesquisadores. Existem muitas pesquisas sobre práticas de ensino com base nas contribuições da Análise do Comportamento (Henklain \& Carmo, 2013), entretanto são trabalhos sobre princípios gerais relacionados ao ensino. Eles se aplicam ao ensino de Matemática, como de qualquer outra disciplina. O que se está sugerindo é que sejam conduzidos estudos para tratar de necessidades específicas ao ensino de Matemática. Ainda que uma abordagem de aplicação de princípios gerais de ensino seja altamente valiosa, pertinente e mais útil (porque é mais geral e aplicável a mais casos), é preciso reconhecer que cada conteúdo tem as suas especificidades e requer, portanto, estudo e preparo para que possa ser ensinado com maior eficácia.

As dificuldades no ensino de Matemática justificam o esforço de se conduzir estudos es- pecíficos nessa área. Na presente pesquisa, verificou-se que estudos sobre o comportamento de ensinar Matemática têm sido desenvolvidos na UNESP de Bauru sob a orientação do professor Jair Lopes Júnior - conduzidos por pesquisadores, tais como Deise A. P. Sparvoli, Daniela C. Maestro, Jurandyr de Oliveira e Aila S. de Almeida - e que muito se discute e produz acerca de como ensinar melhor com base nos trabalhos de Carolina Bori e Sílvio Paulo Botomé, tendo eles próprios orientado trabalhos sobre ensino de Matemática.

Os dados do presente estudo sugerem que muitos trabalhos de mestrado têm sido produzidos sobre ensino-aprendizagem da Matemática e Análise do Comportamento. Algumas possibilidades para o pequeno número de teses (15) são: as pesquisas iniciadas no mestrado não tiveram continuidade no doutorado, os pesquisadores optaram por mudar o seu tema de pesquisa ou não fizeram doutorado, ou ainda, o motivo é que muitos programas de pós-graduação brasileiros ainda não possuem cursos de doutorado e nem todos tem orientadores analistas do comportamento. Sabe-se que as pesquisas de mestrado, pelas variáveis tempo (cerca de 2 anos) e inexperiência do pesquisador, sofrem limitações, tais como o número de estudos que se pode realizar. Em alguns programas de mestrado como o de Psicologia da UFSCar, por exemplo, quase 1 ano é dedicado a elaboração do projeto, aprovação pelo Comitê de Ética e participação em disciplinas. $\mathrm{O}$ ano seguinte deve ser destinado à pesquisa, exames de qualificação e defesa, redação da dissertação, participação em congressos, etc., num contexto em que tudo tende a ser novidade. O tempo do doutorado aliado à maior experiência do pesquisador, geram condições mais apropriadas para o aprofundamento num tema de pesquisa e para a inovação. Se as pesquisas sobre CM e CEM são descontinuadas, é preciso que se entenda o porquê e que se estimulem mais pesquisas analítico-comportamentais no nível de doutorado sobre ensino-aprendizagem da Matemática.

Os dados do presente estudo indicam que existe uma concentração da produção nacional na região sudeste do país, especificamente, no 
estado de São Paulo: PUC-SP, USP e UNESP respondem por 36 trabalhos; junto com a UFSCar alcançam o patamar de $81,37 \%$ da produção nacional na área, sem considerar outras instituições que foram colocadas na categoria "outras" e também são do estado de São Paulo. Para quem busca, portanto, formação e interações com pesquisadores da área, uma sugestão é buscar os programas de pós-graduação do estado de São Paulo. Essa concentração tende a não ser positiva, pelo menos, em dois sentidos: (a) existem realidades locais do ensino de Matemática que não estão sendo estudadas e (b) é provável que a transferência de conhecimento, sob a forma de projetos de extensão, da universidade para a sociedade nessa linha de pesquisa também esteja concentrada nesses poucos centros.

Não é habitual nos cursos de graduação em Psicologia, disciplinas exclusivas ou que abordem contribuições analítico-comportamentais ao ensino-aprendizagem da Matemática. Logo, a concentração da produção sugere que poucos psicólogos têm contato com esse conhecimento, de modo que terão de buscar sozinhos por essa formação. Caso não o façam, não serão disseminadores das contribuições analítico-comportamentais ao ensino-aprendizagem da Matemática. Por outro lado, o número expressivo de apresentações de trabalho sobre ensino-aprendizagem da Matemática em eventos científicos, oriundos de diferentes instituições, sugere que pesquisas sobre o tema estão ocorrendo em todo o país e que existe o interesse de alunos e de pesquisadores. Essas pesquisas deveriam ser desenvolvidas no âmbito da pós-graduação justamente pelo que se discutiu anteriormente acerca da disponibilidade de esforços do pesquisador para aprofundar-se no tema.

Os dados obtidos neste estudo sobre instituições e pesquisadores permitem identificar as pessoas por trás das instituições. Em futuras pesquisas de levantamento sobre as contribuições comportamentais ao ensino-aprendizagem da Matemática, são esses os nomes de pesquisadores que podem ser entrevistados ou cujos currículos Lattes podem ser analisados nessa tarefa de mapear a produção comportamental na área.

\section{Considerações Finais}

Os desafios e a importância do ensino-aprendizagem da Matemática associados à relevância das contribuições da Análise do Comportamento para o ensino e para a compreensão dos processos psicológicos envolvidos no aprendizado da Matemática justificam o esforço de mapear e sistematizar o conhecimento produzido. Nesta revisão bibliográfica verificou-se que na produção dos analistas do comportamento sobre ensino-aprendizagem da Matemática há priorização de estudos sobre o comportamento matemático e a necessidade, portanto, de mais contribuições específicas sobre o comportamento de ensinar Matemática, bem como a disseminação das pesquisas analítico-comportamentais sobre ensino-aprendizagem da Matemática pelo Brasil. Isso é relevante para gerar debates na graduação e estímulo para a realização de estudos em nível de pós-graduação. Seguindo as contribuições de Del Rey (2009), o enfoque deste trabalho foi sobre a produção nacional, embora novas pesquisas devam ampliar o escopo deste levantamento para a produção analítico-comportamental sobre ensino-aprendizagem da Matemática em outros países. Sugere-se que as próximas pesquisas realizem duas tarefas: (a) mapear artigos nacionais e/ou internacionais de base analítico-comportamental sobre ensino-aprendizagem da Matemática e (b) criar mais categorias de análise da produção, separando, por exemplo, pesquisas sobre "ansiedade à Matemática" de pesquisas sobre "ensino de resolução de problemas" e, também, identificar contribuições comportamentais em termos dos ramos da Matemática, tais como álgebra, aritmética, geometria e assim por diante.

\section{Referências}

Araújo, C. A. (2006). Bibliometria: Evolução histórica e questões atuais. Educação \& Sociedade, 12(1), 11-32.

Araújo, C. H., \& Luzio, N. (2005). Avaliação da Educação Básica: Em busca da qualidade e equidade no Brasil. Brasília, DF: Ministério da Educação. 
Carmo, J. S., \& Prado, P. S. T. (2004). Análise do comportamento e psicologia da educação Matemática: Algumas aproximações. In M. M. C. Hübner \& M. Marinotti (Eds.), Análise do comportamento para a educação: Contribuições recentes (pp. 115-136). Santo André, SP: ESETec.

Critchfield, T. S., \& Fienup, D. M. (2010). Using stimulus equivalence technology to teach statistical inference in a group setting. Journal of Applied Behavior Analysis, 43(4), 763-768. doi:10.1901/ jaba.2010.43-763

Cruz, R. N. (2006). História e historiografia da ciência: Considerações para pesquisa histórica em análise do comportamento. Revista Brasileira de Terapia Comportamental e Cognitiva, 8(2), 161-178.

De Paula, J. B. C. (2009). Pesquisas empíricas com humanos sobre relações de equivalência: Análise de dissertações e teses defendidas no Brasil entre 1998 e 2007 (Dissertação de mestrado, Universidade Estadual de Londrina, PR, Brasil).

Del Rey, D. (2009). Análise do comportamento no Brasil: O que já foi pesquisado até 2005 em relação aos comportamentos matemáticos (Dissertação de mestrado, Pontifícia Universidade Católica de São Paulo, SP, Brasil).

Escobal, G., Rossit, R., \& Goyos, A. C. N. (2010). Aquisição de conceito de número por pessoas com deficiência intelectual. Psicologia em Estudo, 15, 467-475. doi:10.1590/S141373722010000300004

Ferreira, N. S. A. (2002). As pesquisas denominadas “estado da arte". Educação \& Sociedade, 12(1), 11-32. doi:10.1590/S0101-73302002000300013

Fienup, D. M., \& Critchfield, T. S. (2011). Transportability of equivalence-based programmed instruction: Efficacy and efficiency in a college classroom. Journal of Applied Behavior Analysis, 3(44), 435-450. doi:10.1901/jaba.2011.44435

Fiorentini, D. (1994). Rumos da pesquisa brasileira em Educação Matemática: O caso da produção científica em cursos de pós-graduação (Tese de doutorado, Universidade Estadual de Campinas, SP, Brasil).

Henklain, M. H. O., \& Carmo, J. S. (2011). Produção analítico-comportamental sobre ensino-aprendizagem de habilidades matemáticas: Dados representativos de eventos científicos brasileiros.
Perspectivas em Análise do Comportamento, 2(2), 179-191.

Henklain, M. H. O., \& Carmo, J. S. (2013). Contribuições da análise do comportamento à educação: Um convite ao diálogo. Cadernos de Pesquisa, 43(149), 704-723. doi:10.1590/ S0100-15742013000200016.

Iégas, A. L. de F., \& Haydu, V. B. (2015). Resolução de problemas aritméticos: efeito de ensino com o modelo de uma balança virtual. Temas em Psicologia, 23(1), 83-96. doi:10.9788/TP2015.1-06

Levingston, H. B., Neef, N. A., \& Cihon, T. M. (2009). The effects of teaching precurrent behaviors on children's solution of multiplication and division word problems. Journal of Applied Behavior Analysis, 42(2), 361-367. doi:10.1901/ jaba.2009.42-361

Lynch, D. C., \& Cuvo, A. J. (1995). Stimulus equivalence instruction of fraction-decimal relations. Journal of Applied Behavior Analysis, 28, 115126. doi:10.1901/jaba.1995.28-115

Mayfield, K. H., \& Chase, P. N. (2002). The effects of cumulative practice on mathematics problem solving. Journal of Applied Behavior Analysis, 35(2), 105-123. doi:10.1901/jaba.2002.35-105

Mendes, A. C., \& Carmo, J. S. (2014). Atribuições dadas à Matemática e ansiedade ante a Matemática: O relato de alguns estudantes do ensino fundamental. Bolema, 28(50), 1368-1385. doi:10.1590/1980-4415v28n50a18

Micheletto, N., Guedes, M. C., Pereira, M. E. M. P., \& Silva, C. P. (2008). Banco de dados de dissertações e teses em Análise do Comportamento no Brasil 1968-2007 (BTAC). São Paulo, SP: Centro de Referências do Behaviorismo Radical, Laboratório de Estudos Históricos em Análise do Comportamento, Programa de Estudos Pós-graduado em Psicologia Experimental, Pontifícia Universidade Católica de São Paulo.

Organization for Economic Cooperation and Development. (2012). Relatório nacional PISA 2012: Resultados brasileiros. Recuperado em http://download.inep.gov.br/acoes_internacionais/pisa/resultados/2014/relatorio_nacional_ pisa_2012_resultados_brasileiros.pdf

Prado, P. S. T., \& de Rose, J. C. (1999). Conceito de número: Uma contribuição da Análise Comportamental da Cognição. Psicologia: Teoria e Pesquisa, 15(3), 227-235. doi:10.1590/S010237721999000300006 
Resnick, L. B., Wang, M. C., \& Kaplan, J. (1973). Task analysis in curriculum design: A hierarchically sequenced introductory mathematics curriculum. Journal of Applied Behavior Analysis, 6(4), 679-710.

Rossit, R. A. S. (2003). Matemática para deficientes mentais: Contribuições do paradigma de equivalência de estímulos para o desenvolvimento e avaliação de um currículo (Tese de doutorado não publicada, Universidade Federal de São Carlos, SP, Brasil).

Santos, A. C. G., Cameschi, C. E., \& Hanna, E. S. (2012). Ensino de frações utilizando o paradigma de equivalência de estímulos. Revista Brasileira de Análise do Comportamento, 5, 19-41.

Santos, A. C. G., Simonassi, L. E., Rodrigues, C. F., \& Magri, M. R. (2014). Efeito do treino de composição (cópia) na aprendizagem do conceito de proporção. Psicologia: Teoria e Pesquisa, 30, $459-469$
Saville, B. K., Lambert, T., \& Robertson, S. (2011). Interteaching: Bringing behavioral education into de 21 st century. The Psychological Record, 61,153-166.

Skinner, B. F. (1972). Tecnologia do ensino (R. Azzi, Trans.). São Paulo, SP: Herder. (Original publicado em 1968)

Teixeira, A. M. S. (2006). Análise de contingências em programação de ensino infantil: Liberdade e efetividade na educação. Santo André, SP: ESETec. 